\title{
Monodisperse liquid crystalline polymer shells with programmable alignment and shape prepared by seeded dispersion polymerization
}

\author{
Citation for published version (APA): \\ Liu, X., Moradi, M., Bus, A. B. P., Heuts, J. P. A., Debije, M. G., \& Schenning, A. P. H. J. (2021). Monodisperse \\ liquid crystalline polymer shells with programmable alignment and shape prepared by seeded dispersion \\ polymerization. Macromolecules, 54(13), 6052-6060. https://doi.org/10.1021/acs.macromol.1c00884
}

Document license:

CC BY

DOI:

10.1021/acs.macromol.1c00884

Document status and date:

Published: $13 / 07 / 2021$

Document Version:

Publisher's PDF, also known as Version of Record (includes final page, issue and volume numbers)

\section{Please check the document version of this publication:}

- A submitted manuscript is the version of the article upon submission and before peer-review. There can be important differences between the submitted version and the official published version of record. People interested in the research are advised to contact the author for the final version of the publication, or visit the $\mathrm{DOI}$ to the publisher's website.

- The final author version and the galley proof are versions of the publication after peer review.

- The final published version features the final layout of the paper including the volume, issue and page numbers.

Link to publication

\footnotetext{
General rights

- You may freely distribute the URL identifying the publication in the public portal. follow below link for the End User Agreement:

www.tue.nl/taverne

Take down policy

If you believe that this document breaches copyright please contact us at:

openaccess@tue.nl

providing details and we will investigate your claim.
}

Copyright and moral rights for the publications made accessible in the public portal are retained by the authors and/or other copyright owners and it is a condition of accessing publications that users recognise and abide by the legal requirements associated with these rights.

- Users may download and print one copy of any publication from the public portal for the purpose of private study or research.

- You may not further distribute the material or use it for any profit-making activity or commercial gain

If the publication is distributed under the terms of Article $25 \mathrm{fa}$ of the Dutch Copyright Act, indicated by the "Taverne" license above, please 


\title{
Monodisperse Liquid Crystalline Polymer Shells with Programmable Alignment and Shape Prepared by Seeded Dispersion Polymerization
}

\author{
Xiaohong Liu, Mohammad-Amin Moradi, Tom Bus, Johan P.A. Heuts, Michael G. Debije, \\ and Albert P.H.J. Schenning*
}

Cite This: Macromolecules 2021, 54, 6052-6060

Read Online

ACCESS | Lلll Metrics \& More | 国 Article Recommendations | s supporting Information

ABSTRACT: Monodisperse, micrometer-sized liquid crystalline (LC) shells are prepared by seeded dispersion polymerization. After polymerizing LC monomer mixtures in the presence of noncrosslinked polymer seeds, hollow LC polymer shells with programmable alignment and shape are prepared by removing the seeds. The LC alignment in the LC polymer shells can be easily manipulated by the polymer seeds, as a radial alignment is observed with amorphous poly(phenyl methacrylate) seeds and a bipolar alignment is observed with bipolar LC polymer seeds. After removal of the seeds, the radially aligned samples give radially

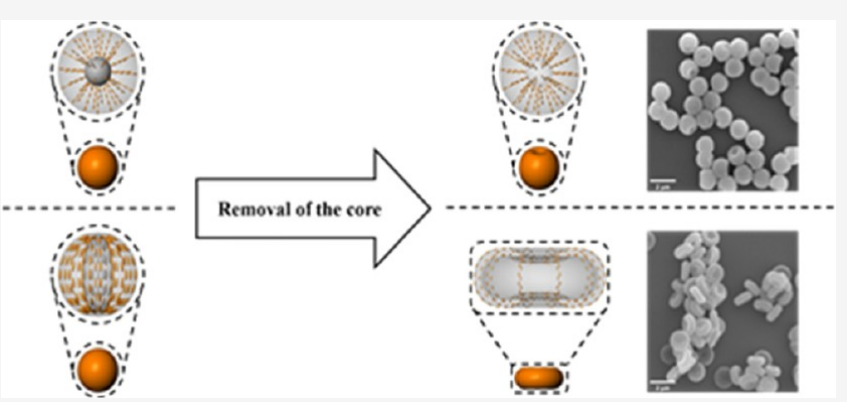
aligned shells with small dimples. The resulting bipolar LC polymer shells collapse into a biconcave shape. Polarized optical microscopy and transmission electron microscopy indicate that the collapse occurs at the defect points in the shell. In the case of a lower crosslink density, LC polymer hollow shells with larger dimples are obtained, resulting in cup-shaped polymer particles. Biconcave LC polymer shells based on other LC mixtures have also been prepared, showing the versatility of the seeded dispersion polymerization method.

\section{INTRODUCTION}

Polymer shells are of interest for use in drug delivery, ${ }^{1-3}$ in separation of chiral mixtures, ${ }^{4-7}$ and as a host for catalysts. ${ }^{8-10}$ Among hollow particles, liquid crystalline (LC) polymer shells are particularly interesting, as the alignment of LC molecules gives rise to novel anisotropic functional properties. ${ }^{11-14}$ Monodisperse LC polymer shells have been prepared using microfluidics; ${ }^{11-14}$ however, preparing well-defined LC polymer shells with tunable alignment with dimensions smaller than $10 \mu \mathrm{m}$ remains a challenge.

Requiring only temperature control and mild agitation, dispersion and precipitation polymerizations have emerged as promising alternatives for preparing monodisperse LC polymer particles with average diameters ranging from a few micrometers to less than $1 \mu \mathrm{m} \cdot{ }^{15-17}$ Based on these polymerization methods, seeded dispersion polymerization has been developed for amorphous polymers. ${ }^{18,19}$ In this process, polymerization commences in the presence of preexisting seeds with the newly-formed polymer chains adsorbing onto the surface of the seeds, resulting in a distinctive core-shell structure. The technique offers great versatility in chemical composition, and by selectively removing the inner core, hollow polymer particles can be prepared. This method has been widely used to prepare amorphous particles, but to the best of our knowledge, ordered LC polymer shells prepared by seeded dispersion polymerization have never been reported.
Herein, we report the facile preparation of monodisperse micrometer-sized LC polymer shells with programmed alignment and shape via seeded dispersion polymerization. Since the outer surface of the seed functions as an alignment layer for the LC molecules making up the shell, we expected differing alignments in the shell dependent on whether amorphous poly(phenyl methacrylate) (PPhMA) or LC bipolar polymer particles were used as seeds. In what follows, we show that the nature of the seed indeed determines the alignment and shape of the monodisperse, micrometer-sized shell, which consists of a combination of the acrylic LC monomers as shown in Figure 1.

\section{EXPERIMENTAL SECTION}

2.1. Materials. 4-Methoxyphenyl 4-((6-(acryloyloxy)hexyl)oxy)benzoate (1), 2-methyl-1,4-phenylene bis(4-((6-(acryloyloxy)hexyl)oxy)benzoate) (2), 4,4'-bis(6-acryloyloxyhexyloxy) azobenzene (3), and 4-(6-acryloxyhexyl-1-oxy)benzoic acid (4) (see Figure 1) were purchased from Synthon Chemicals, Germany. Phenyl methacrylate

Received: April 23, 2021

Revised: June 4, 2021

Published: June 22, 2021 

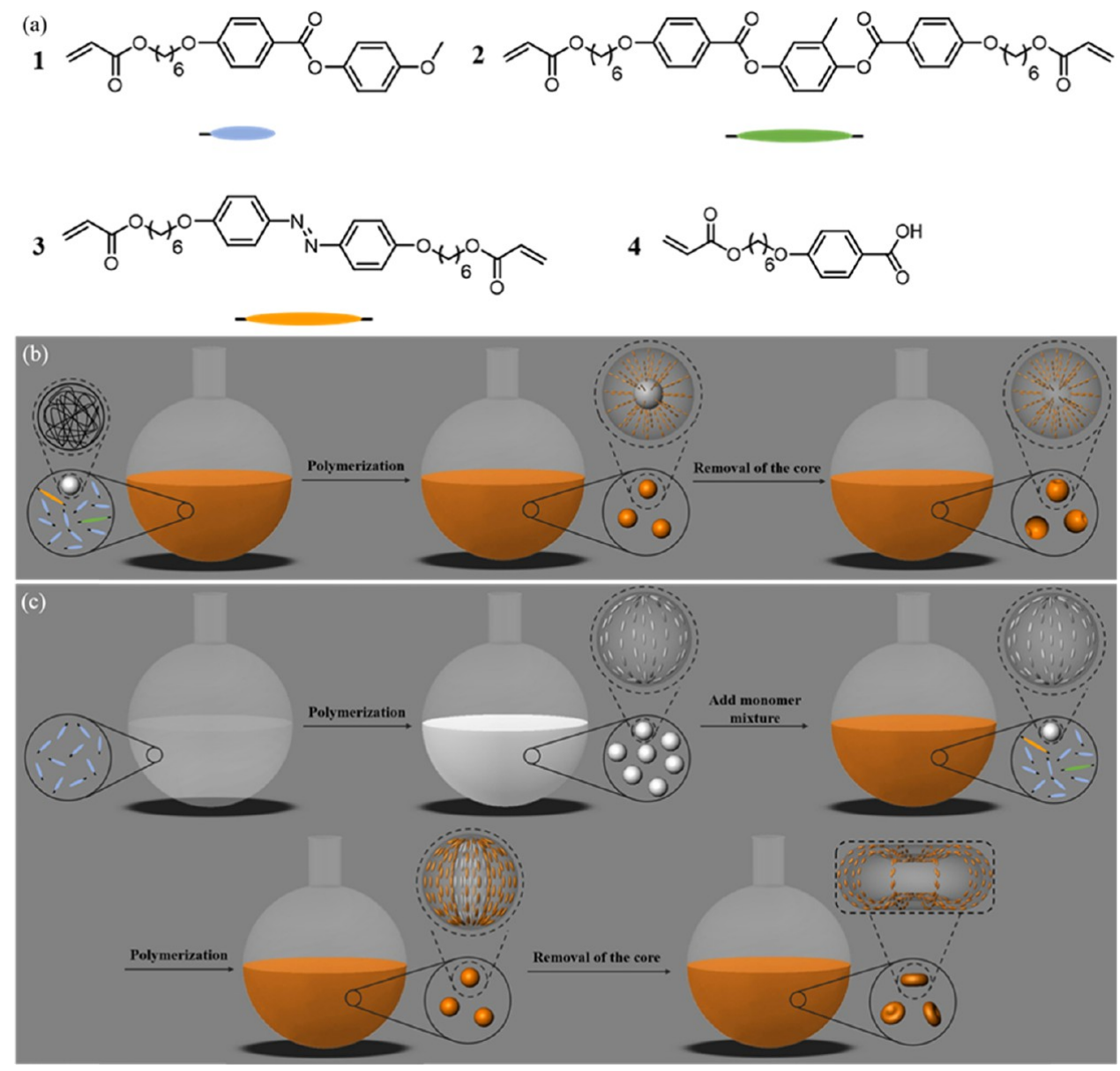

Figure 1. (a) Monomers employed to prepare the LC polymer shells. (b) Schematic representation of the synthesis of LC shells with radial alignment using amorphous PPhMA seeds. (c) Schematic representation of the synthesis of LC shells with bipolar alignment using bipolar LC seeds.

(PhMA, 97\%) was purchased from TCI Europe. Polyvinyl pyrrolidone (PVP K30) and 2,2'-azobis(2-methylpropionitrile) (AIBN, 98\%) were purchased from Sigma-Aldrich. All solvents were purchased from Biosolve.

2.2. Preparation of LC Shells via Seeded Dispersion Polymerization. 2.2.1. Synthesis of Core-Shell LC Particles with Amorphous PPhMA Seeds. Poly(phenyl methacrylate) (PPhMA) seeds were prepared by dispersion polymerization. To this end,100 $\mathrm{mg}$ of PVP and $10 \mathrm{mg}$ of AIBN were added to a $50 \mathrm{~mL}$ round-bottom flask, which was then evacuated and backfilled with nitrogen 3 times. Subsequently, $1 \mathrm{~mL}$ of PhMA dissolved in $9 \mathrm{~mL}$ of ethanol was added to the flask, after which the flask was put into an oil bath preheated to $70{ }^{\circ} \mathrm{C}$ and stirred at $200 \mathrm{rpm}$ under $\mathrm{N}_{2}$ overnight. After the polymerization, the PPhMA particles were centrifuged and washed with ethanol three times. Yield $=77 \%$.

The PPhMA seed particles were then used to prepare core-shell LC particles. To a $50 \mathrm{~mL}$ round-bottom flask, $90 \mathrm{mg}$ of monomer 1,5 $\mathrm{mg}$ of monomer $2,5 \mathrm{mg}$ of monomer $3,20 \mathrm{mg}$ of PVP, and $2 \mathrm{mg}$ of AIBN were added, after which the round-bottom flask was then pumped and backfilled with nitrogen 3 times. Subsequently, $5 \mathrm{~mL}$ of ethanol was added and the round-bottom flask was put into an oil bath preheated to $70{ }^{\circ} \mathrm{C}$. Upon complete dissolution of the monomers, $100 \mathrm{mg}$ of PPhMA particles was dispersed in $5 \mathrm{~mL}$ of ethanol and added. The polymerization was carried out at $70{ }^{\circ} \mathrm{C}$ and stirred at $200 \mathrm{rpm}$ under $\mathrm{N}_{2}$ overnight. After polymerization, the dispersion was centrifuged and washed with ethanol to obtain the particles. The particles were stored in $5 \mathrm{~mL}$ of ethanol prior to use, and the solid content was accurately measured by drying $50 \mu \mathrm{L}$ of suspension in a DSC pan. Yield $=88 \%$, where yield is defined as the weight of the core-shell LC particles after seeded dispersion polymerization divided by the total weight of LC monomers and PPhMA seeds.

2.2.2. Synthesis of Core-Shell LC Particles with LC Seeds. First, the LC seed particles were synthesized via dispersion polymerization. To a $50 \mathrm{~mL}$ round-bottom flask, $100 \mathrm{mg}$ of monomer $1,10 \mathrm{mg}$ of PVP, and $2 \mathrm{mg}$ of AIBN were added, after which the flask was pumped and backfilled with nitrogen 3 times. Subsequently, $5 \mathrm{~mL}$ of ethanol was added to the flask, which was then put into an oil bath preheated to $70{ }^{\circ} \mathrm{C}$ and stirred at $200 \mathrm{rpm}$ under $\mathrm{N}_{2}$ for $2 \mathrm{~h}$. Without isolating the seed particles, $60 \mathrm{mg}$ of monomer $1,5 \mathrm{mg}$ of monomer 2 , $5 \mathrm{mg}$ of monomer 3 , and $10 \mathrm{mg}$ of PVP were dissolved in $5 \mathrm{~mL}$ of hot ethanol and added to the reaction mixture in the flask. The polymerization was carried out overnight. After polymerization, the dispersion was centrifuged and washed with ethanol to obtain the particles. The particles were stored in $5 \mathrm{~mL}$ of ethanol prior to use, and the solid content was accurately measured by drying $50 \mu \mathrm{L}$ of suspension in a DSC pan. Yield $=73 \%$, where yield is defined as the weight of the core-shell particles after seeded dispersion polymerization divided by the total weight of LC monomers.

2.2.3. Synthesis of Carboxylic Acid-Functionalized Core-Shell LC Particles with LC Seeds. Carboxylic acid-functionalized core-shell LC particles were prepared in a similar manner as described in 2.2.2. First, the LC seed was prepared by adding $100 \mathrm{mg}$ of monomer 1, 10 $\mathrm{mg}$ of PVP, and $2 \mathrm{mg}$ of AIBN to a $50 \mathrm{~mL}$ round-bottom flask, pumping and backfilling the flask with nitrogen 3 times, adding $5 \mathrm{~mL}$ of ethanol to the flask and reacting it for $2 \mathrm{~h}$ under $\mathrm{N}_{2}$, and continuous stirring at $200 \mathrm{rpm}$ at $70{ }^{\circ} \mathrm{C}$ in an oil bath. Again, without isolating the seeds, $35 \mathrm{mg}$ of monomer $1,15 \mathrm{mg}$ of monomer 2,20 $\mathrm{mg}$ of carboxylic acid monomer 4 , and $40 \mathrm{mg}$ of PVP were dissolved in $5 \mathrm{~mL}$ of hot ethanol and added to the flask. The polymerization 
was carried out for another $4 \mathrm{~h}$. After polymerization, the dispersion was centrifuged and washed with ethanol to obtain the particles. The core-shell particles were stored in $5 \mathrm{~mL}$ of ethanol prior to use, and the solid content was accurately measured by drying $50 \mu \mathrm{L}$ of suspension in a DSC pan. Yield $=61 \%$, where yield is defined as the weight of the core-shell particles after seeded dispersion polymerization divided by the total weight of LC monomers.

2.2.4. Removal of the Seeds. The particle suspension $(1 \mathrm{~mL})$ of predetermined solid content was added to $9 \mathrm{~mL}$ of tetrahydrofuran (THF), and the particles were allowed to sediment. The solvent was then poured out, and fresh THF was added. This process was repeated 3 times in total. Then, $10 \mathrm{~mL}$ of ethanol was added dropwise with agitation and the particles were allowed to sediment. The solvent was poured out, and $10 \mathrm{~mL}$ of fresh ethanol was added dropwise with agitation. This process was repeated 3 times in total. The LC polymer shells were stored in $5 \mathrm{~mL}$ of ethanol prior to use, and the solid content was accurately measured by drying $50 \mu \mathrm{L}$ of suspension in a DSC pan. Weight loss = 52\% (entry 1), 63\% (entry 3 ), and 36\% (entry 5), where weight loss $=1-$ (weight of THF-treated particles/ weight of initial particles); the weights were calculated from the solid content and the volume of the corresponding suspensions.

2.2.5. Photoresponsiveness of the Bipolar LC Polymer Shell. LC polymer shells in chlorobenzene $(0.3 \mathrm{mg} / \mathrm{mL})$ were added in a quartz cuvette. The suspension was immediately subjected to UV-vis measurement, denoted as "measurement 0 ." Then, the suspension was irradiated with $365 \mathrm{~nm}$ light for $1 \mathrm{~min}$, and the suspension was subjected to another UV-vis measurement, denoted as "measurement 1." The suspension was irradiated with $455 \mathrm{~nm}$ light for $1 \mathrm{~min}$, and the suspension was subjected to another UV-vis measurement, denoted as "measurement 2." Five cycles of alternative irradiation were performed, and the UV-vis spectra were measured.

2.3. Characterization. Polarized optical microscopy (POM) images were taken with a Leica CTR6000 polarized optical microscope and a Leica DFC 420C camera. POM images were taken by drying the particle suspensions on clean glass slides unless otherwise stated. ${ }^{1} \mathrm{H}$ NMR spectra were recorded on a $400 \mathrm{MHz}$ Bruker Avance III HD spectrometer in deuterated chloroform with tetramethyl silane used as an internal standard. Scanning electron microscopy (SEM) images were taken with a JEOL TM 220 A. The average size and coefficient of variation of the particle size distribution $\left(C_{\mathrm{v}}=\frac{\bar{d}}{\mathrm{SD}}\right)$ were measured using ImageJ. The polymer particles and their cross sections were investigated via transmission electron microscopy (TEM) using a Tecnai 20 (type Sphera) by FEI operating with a LaB 6 filament at $200 \mathrm{kV}$ under slight under-focus conditions. For the cross section, particles were embedded in an EPOFIX epoxy medium. Cross sections were cut at room temperature using an ultramicrotome (Reichert-Jung Ultracut E) with a setting thickness of 60 $\mathrm{nm}$. The obtained cross sections were transferred to a carbon filmcovered grid (Electron Microscopy Sciences, CF200-CU). UV-vis spectra were measured with a Shimadzu UV-3102 PC. Thorlabs light emitting diode lamps with collimation adapters were used to provide 365 and $455 \mathrm{~nm}$ lights. The distance between the lamps and the cuvette was about $30 \mathrm{~cm}$. The light intensities were about $120 \mathrm{~mW} /$ $\mathrm{cm}^{2}(365 \mathrm{~nm})$ and $45 \mathrm{~mW} / \mathrm{cm}^{2}(455 \mathrm{~nm})$.

\section{RESULTS AND DISCUSSION}

\subsection{Synthesis of LC Shells via Seeded Dispersion} Polymerization with PPhMA Seeds. Monodisperse PPhMA particle seeds were first prepared by dispersion polymerization at $70{ }^{\circ} \mathrm{C}$ in ethanol, with PVP as the stabilizer and AIBN as the thermal initiator. SEM images revealed an average diameter of $1.50 \mu \mathrm{m}$ and a coefficient of variation of 3.7\% (Figure 2a). The PPhMA particle seeds were redispersed in ethanol and then added to an ethanol solution containing LC monoacrylate $\mathbf{1}$, LC diacrylate 2 , and azobenzene diacrylate 3 (Table 1, entry 1, Figure 2b), PVP, and AIBN. The suspension was heated to $70{ }^{\circ} \mathrm{C}$ and polymerized overnight,
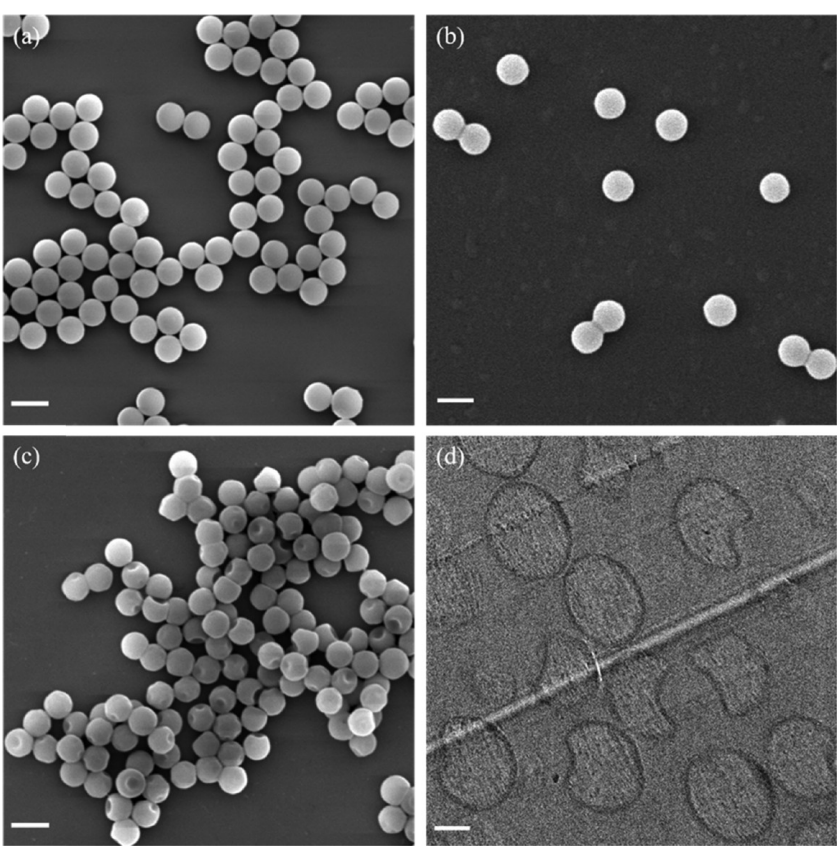

Figure 2. SEM images of (a) PPhMA seeds, (b) core-shell particles after seeded dispersion polymerization, and (c) LC shells after THF treatment (scale bar $(\mathrm{a}-\mathrm{c})=2 \mu \mathrm{m})$; (d) TEM images of the cross section of the LC shells (scale bar $=0.5 \mu \mathrm{m}$ ).

yielding spherical core-shell particles with an average diameter of $1.69 \mu \mathrm{m}$ and a coefficient of variation of $3.8 \%$ (Figure $2 \mathrm{~b}$ ). This indicates a thickness of approximately $100 \mathrm{~nm}$ for the cross-linked shell. To remove the PPhMA core (which consists of linear PPhMA chains), the particles were treated with THF repeatedly, prior to transferring and storing in ethanol. The treatment with THF resulted in a weight reduction of 52\%, which suggests a near complete removal of the PPhMA core; the original composition of the core-shell particles was $56 \mathrm{wt}$ $\%$ core and $44 \mathrm{wt} \%$ shell. SEM images of the LC shells (Figure $2 c$ ) show dimpled particles with an average diameter of about $1.46 \mu \mathrm{m}$. The Hansen solubility parameter of the LC polymer shells was estimated with a group contribution method, ${ }^{20}$ and the solubility distance $\left(R_{\mathrm{a}}\right)$ to ethanol and THF was calculated (Tables S1 to S3). The lower number of $R_{\mathrm{a}}$ to THF suggests that THF has a higher compatibility than ethanol with the LC, and hence, it is conceivable that THF diffusion out of the shell is faster than ethanol diffusion into the shells, resulting in an osmotic pressure that could locally compress the shell. The TEM images of a cross section of the shells (Figure 2d) confirm a thickness of about $100 \mathrm{~nm}$ for the LC shells, which can be seen as the dark rings with dimples; this result is consistent with the increase of the particle diameter after the seeded dispersion polymerization (Figure 2a,b).

To study the LC alignment, the LC shells were dispersed in ethanol and dried on a glass slide for POM. The POM image in Figure 3a without crossed polarizers shows monodisperse, micrometer-sized, almost spherical shells with occasional dimples consistent with the SEM image in Figure 2c. The POM images obtained with crossed polarizers are shown in Figure $3 \mathrm{~b}, \mathrm{c}$. Maltese crosses were always observed whose direction was always parallel to the orientation of one of the crossed polarizers, indicating that the LC molecules in the polymer shells aligned radially, that is, perpendicular to the shell surface, as schematically shown in Figure 3d. 
Table 1. LC Polymer Core-Shell Particles and Hollow Shells Prepared by Seeded Dispersion Polymerization

\begin{tabular}{|c|c|c|c|c|c|}
\hline entry & $\begin{array}{l}\text { monomer } \\
\text { mixture }\end{array}$ & type/diameter of the seeds & diameter of the core-shell particles & diameter/shape of the LC shells & alignment of the LC shells \\
\hline 1 & $1,2,3$ & $\mathrm{PPhMA} / 1.50 \mu \mathrm{m}$ & $1.69 \mu \mathrm{m}$ & $1.46 \mu \mathrm{m} / \mathrm{spheres}$ with small dimples & radial \\
\hline 2 & 1,3 & $\mathrm{PPhMA} / 1.50 \mu \mathrm{m}$ & $1.86 \mu \mathrm{m}$ & N.A. ${ }^{b} /$ cup-shape & radial \\
\hline 3 & $1,2,3$ & bipolar LC polymer seeds ${ }^{a}$ & $1.63 \mu \mathrm{m}$ & $1.59 \mu \mathrm{m} \times 0.61 \mu \mathrm{m} /$ biconcave & bipolar \\
\hline 4 & 1,3 & bipolar LC polymer seeds ${ }^{a}$ & $1.82 \mu \mathrm{m}$ & N.A. ${ }^{b} /$ cup-shape & radial \\
\hline 5 & $1,2,4$ & bipolar LC polymer seeds ${ }^{a}$ & $1.38 \mu \mathrm{m}$ & $1.32 \mu \mathrm{m} \times 0.68 \mu \mathrm{m} /$ biconcave & bipolar \\
\hline
\end{tabular}

${ }^{a}$ No diameters were measured because the seeds were not isolated. ${ }^{b}$ The diameter of the LC shells cannot be measured accurately due to the extensive deformations.
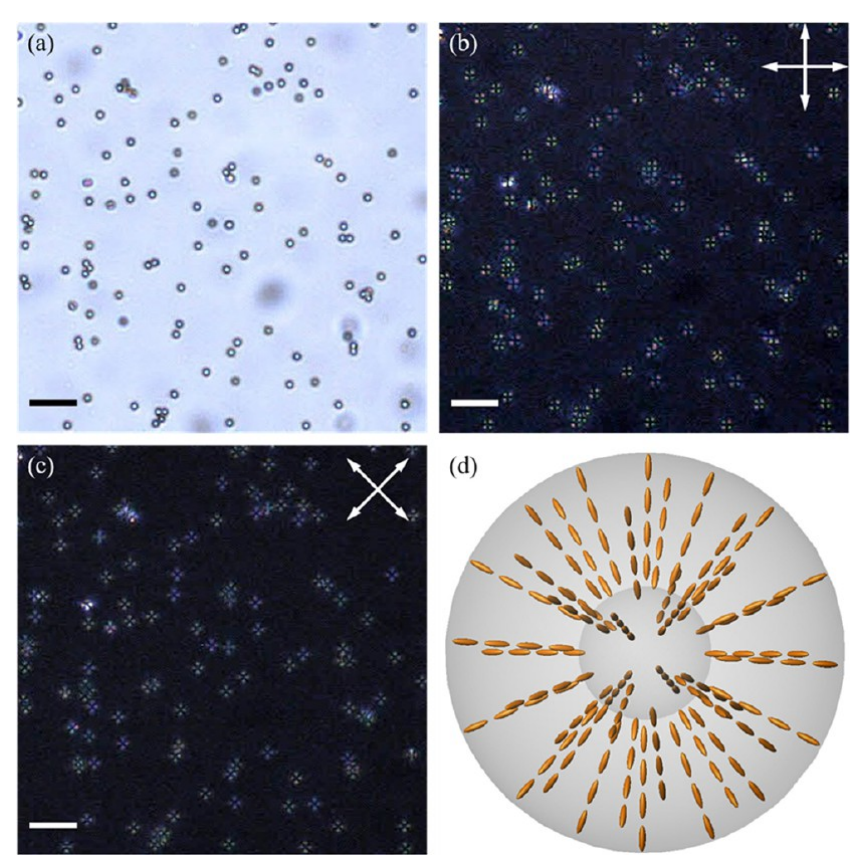

Figure 3. POM images of LC shells (a) without and (b, c) with crossed polarizers (scale bar $=10 \mu \mathrm{m})$ prepared by using PPhMA seeds. The orientation of the crossed polarizers is indicated by the arrows. (d) Schematic representation of the LC alignment in the LC shells using PPhMA seeds.

Seeded dispersion polymerization of a LC monomer mixture consisting of monomers $\mathbf{1}$ and $\mathbf{3}$ was also performed (Table 1, entry 2, and Figure 4). Since there are relatively more monoacrylates in the forming shells than in entry 1 , the final crosslink density in the polymerized shells is likely lower, resulting in hollow shells capable of collapsing and forming larger dimples and cup-shaped polymer particles. Maltese crosses were observed in the POM images, again indicating radial alignment of the LCs in the polymer shells.

3.2. Synthesis of LC Shells via Seeded Dispersion Polymerization with Bipolar LC Seeds. To induce noncentral symmetric alignment in the LC shells, dispersion polymerization of LC monomer 1 developed by Zentel et al. was $\operatorname{adopted}^{15,21}$ to produce noncrosslinked bipolar LC polymer seeds, which were then used as seeds. Upon forming the bipolar LC polymer seeds, the monomer mixture for the LC polymer shell was added to the dispersion without separation of the seeds, and the polymerization was continued to form the core-shell particles. LC monomer 1, PVP, and AIBN were dissolved in ethanol and polymerized at $70{ }^{\circ} \mathrm{C}$ for $2 \mathrm{~h}$ to form the noncrosslinked bipolar LC seeds in situ (Figure 5a). A small volume of suspension was withdrawn from the mixture and subjected to POM and ${ }^{1} \mathrm{H}$ NMR to determine the alignment of these LC particles and the conversion, respectively. The LC particle seeds showed bright spots under crossed polarizers, indicating that the LC molecules in the particles aligned in a bipolar manner, similar to that reported before. ${ }^{15}$ The integration of remaining acrylate peaks at 5.5 to $6.5 \mathrm{ppm}$ in the ${ }^{1} \mathrm{H}$ NMR spectrum indicated that around $30 \%$ of monomer 1 remained unreacted at this point (Figure S1). Therefore, the amount of monomer $\mathbf{1}$ added in the second stage was correspondingly reduced to maintain the composition of the shells identical to the shells made using the PPhMA seeds; the LC monomer mixture was added in the flask without separation and purification of the LC seeds. Seeded dispersion polymerization was continued overnight, yielding core-shell particles with an average diameter of 1.63 $\mu \mathrm{m}$ and a coefficient of variation of $4.3 \%$ (Table 1 , entry 3 , and Figure $5 \mathrm{~b}$ ). The noncrosslinked LC seeds were removed by THF, similar to the removal of the PPhMA seeds. The weight of the LC shells was reduced by $63 \%$, suggesting successful seed removal, as the core and shell initially made up 57 and $43 \%$ of the core-shell particle. SEM images revealed that the remaining hollow shells collapsed into a biconcave shape with two dimples distributed symmetrically at opposite faces of the hollow shells (Figure 5c). While the average diameter of the shells remained almost unchanged as $1.59 \mu \mathrm{m}$, the height of the shell decreased significantly to $0.61 \mu \mathrm{m}$. TEM images further confirmed the size and shape of the LC shells, and the symmetrically distributed dimples were observed as a relatively brighter center upon tilting the sample (Figure $5 \mathrm{~d}$ to $\mathrm{f}$ ).

Maltese crosses were observed parallel to the directions of the cross polarizers after drying the shells on a glass slide (Figure 6a to c). However, since the biconcave shells uniformly "lie down" on the glass slide, meaning that the radii of the spheroids are coplanar with the glass slide surface, in order to investigate the birefringence from different angles, the shells were suspended in glycerol and filled in a glass cell for POM (Figure $6 \mathrm{~d}$ to $\mathrm{f}$ ). Due to the flow of the solvent, LC shells first rotated and flowed (see Movie S1), and as a result, the shape and birefringence pattern of the LC shells continuously changed. Static POM images were taken when the shells stopped moving, and some shells showed dark lines parallel to the direction of the collapse rather than the Maltese cross. From the POM images, it was concluded that the LC shells had a bipolar alignment, and the collapse occurred at the defects, which is probably because the unaligned defects have a lower modulus than the aligned regions (Figure $6 \mathrm{~g}$ ).

LC shells prepared with bipolar LC seeds and a LC monomer mixture with a lower crosslink density (Table 1, entry 4, Figure 7) showed cup-shaped particles with large dimples, similar to the shells prepared with PPhMA seeds using the same LC mixture (Table 1, entry 2, Figure 4). Moreover, Maltese crosses were observed in POM images, 

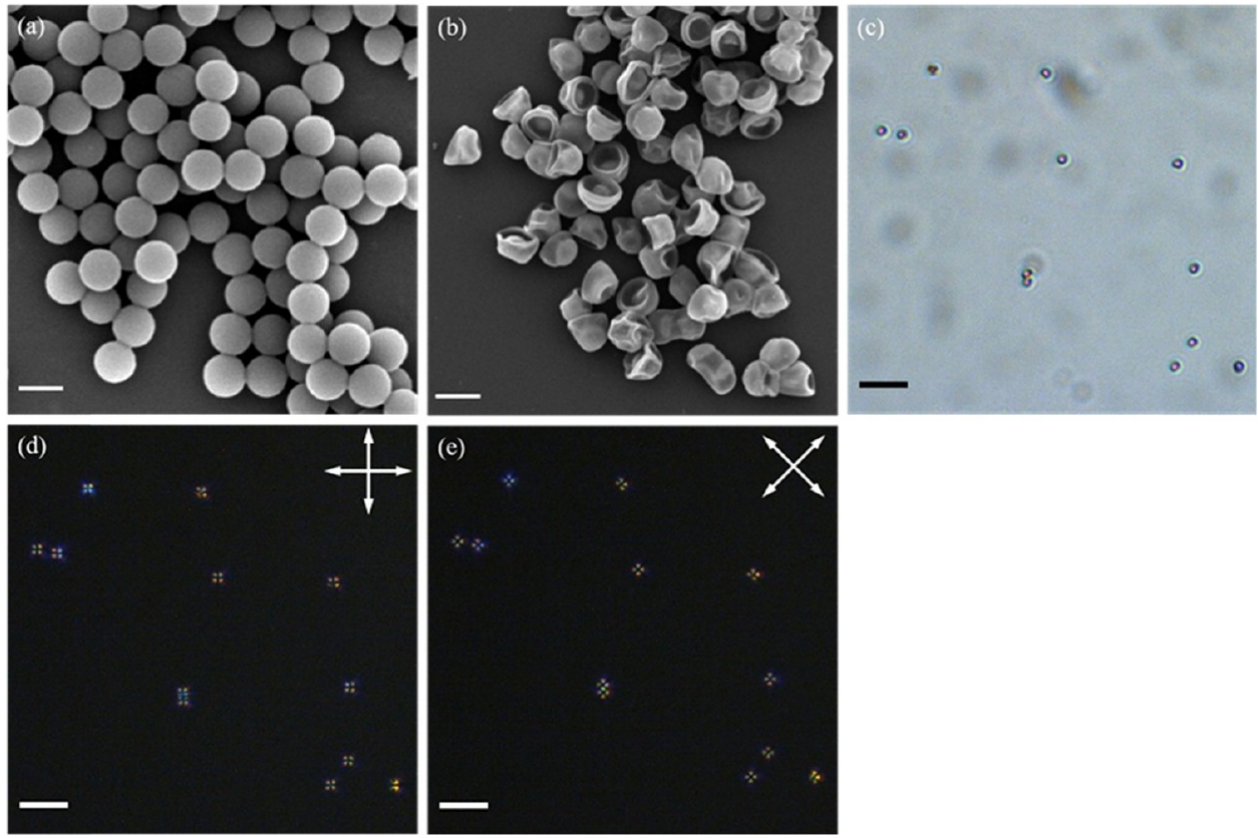

Figure 4. SEM images of (a) core-shell particles and (b) LC shells (scale bar $=2 \mu \mathrm{m}$ ). POM images of LC shells (c) without and (d and e) with cross polarizers (scale bar $=10 \mu \mathrm{m}$ ). The orientation of the cross polarizers is indicated by the arrows.

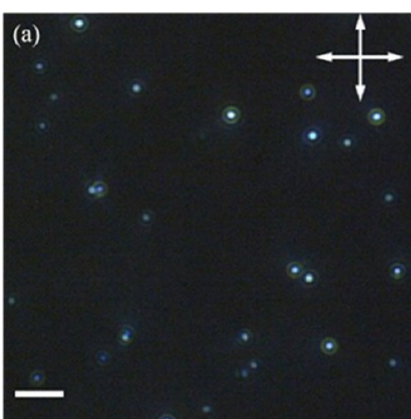

(d)

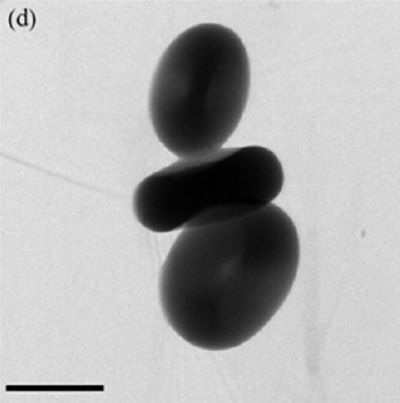

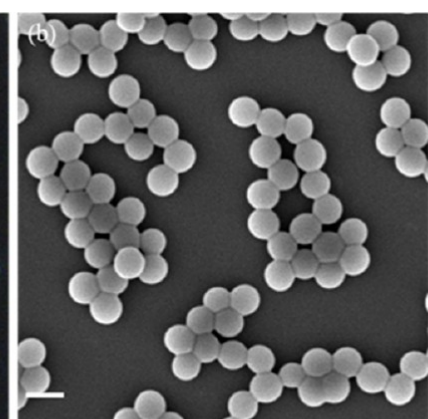

(e)
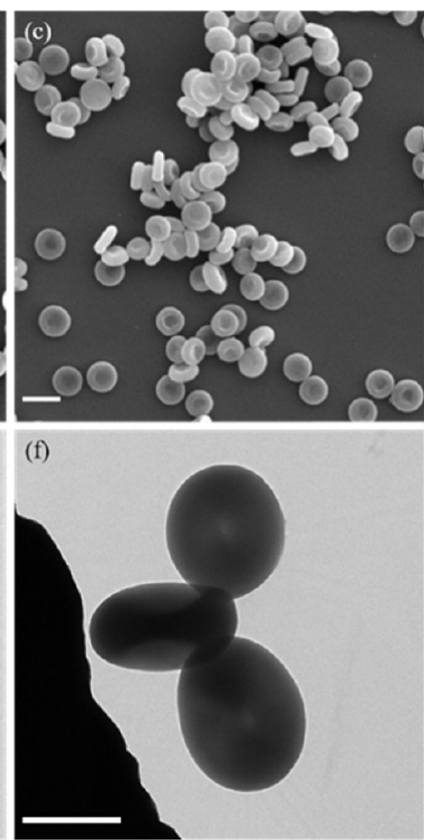

Figure 5. (a) POM images of noncrosslinked LC seeds prepared with monomer 1 with polarizers (scale bar $=10 \mu \mathrm{m})$; SEM images of (b) coreshell particles after seeded dispersion polymerization and (c) hollow LC shells after removing the polymer seeds (scale bar $=2 \mu \mathrm{m})$; (d) to (f) tilt series TEM image of LC shells from different angles (scale bar $=1 \mu \mathrm{m})$.

indicating that the LC molecules aligned radially in the shells, even with bipolar LC seeds. This result suggests that an appropriate crosslink density is necessary to obtain the bipolar alignment in the shells.

\subsection{Seeded Dispersion Polymerization of Other LC}

Mixtures. Seeded dispersion polymerization was also performed using a nematic LC mixture containing monomers 1, 2, and 4 (weight ratio $=65 / 15 / 20$, Table 1 , entry 5). Monomer 4 (Figure 1a) is chosen to investigate the versatility of this seeded dispersion polymerization approach, since carboxylic acid-functionalized monomers have been known to significantly undermine the colloidal stability and make dispersion polymerization challenging. ${ }^{22}$ The in situ preparation of the bipolar LC seeds of monomer 1 was performed as previously described, but since carboxylic acid groups significantly undermine the colloidal stability and are known to make dispersion polymerization challenging, ${ }^{22}$ the second stage of the seeded dispersion polymerization was modified, with the amount of PVP increased and the polymerization time reduced to $4 \mathrm{~h}$. SEM images showed that the average diameter of the resulting core-shell particles was $1.38 \mu \mathrm{m}$ and the coefficient of variation was $8.1 \%$ (Figure $8 \mathrm{a}$ ), indicating that 

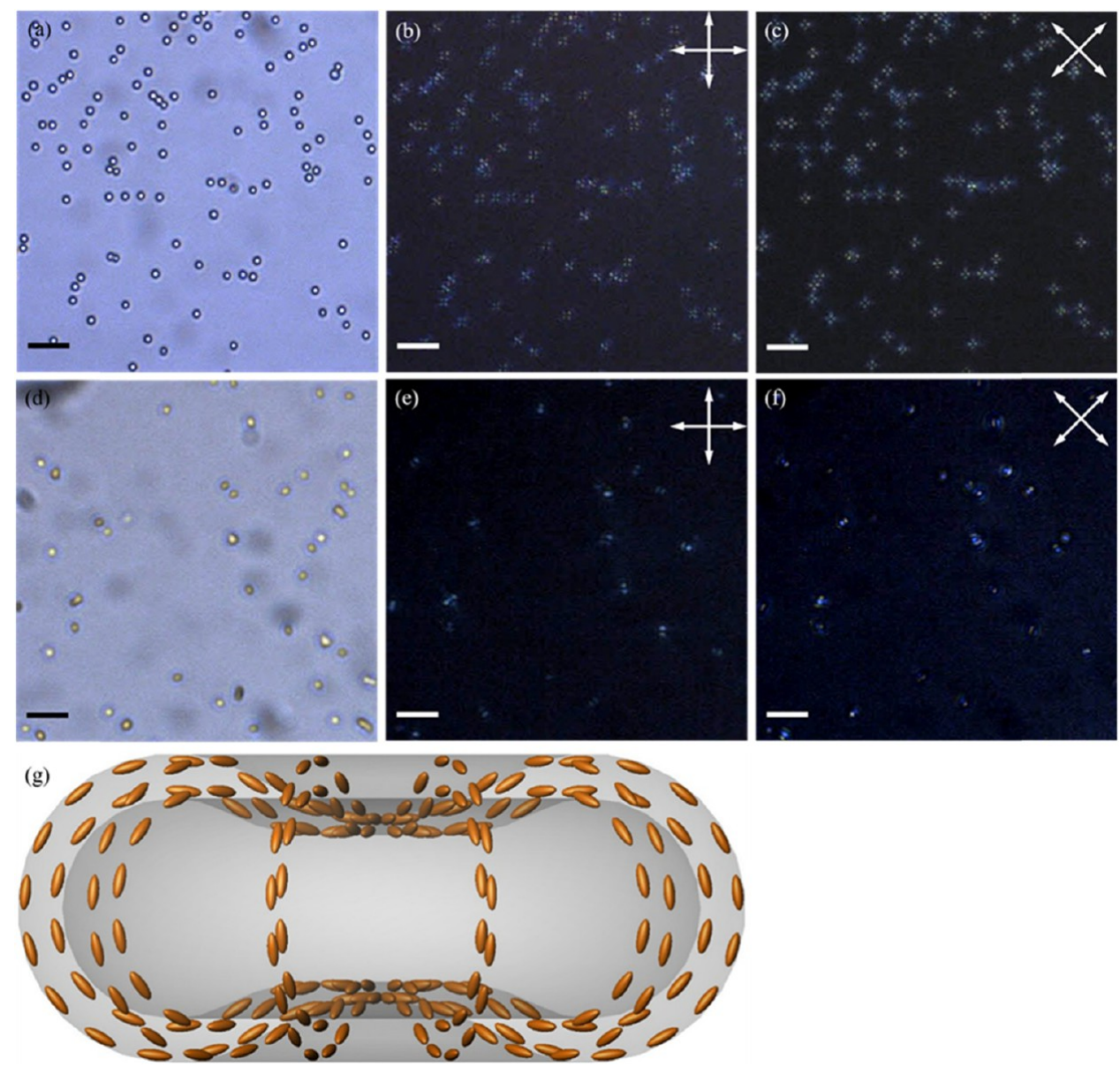

Figure 6. POM images of the LC shells (a) without and ( $\mathrm{b}$ and $\mathrm{c}$ ) with crossed polarizers (scale bar $=10 \mu \mathrm{m}$ ) prepared by using bipolar LC seeds. POM images of the LC shells suspended in glycerol (d) without and (e and f) with crossed polarizers (scale bar $=10 \mu \mathrm{m}$ ). The orientation of the crossed polarizers is indicated by the arrows. $(\mathrm{g})$ Schematic representation of the structure and LC alignment of the LC shells.
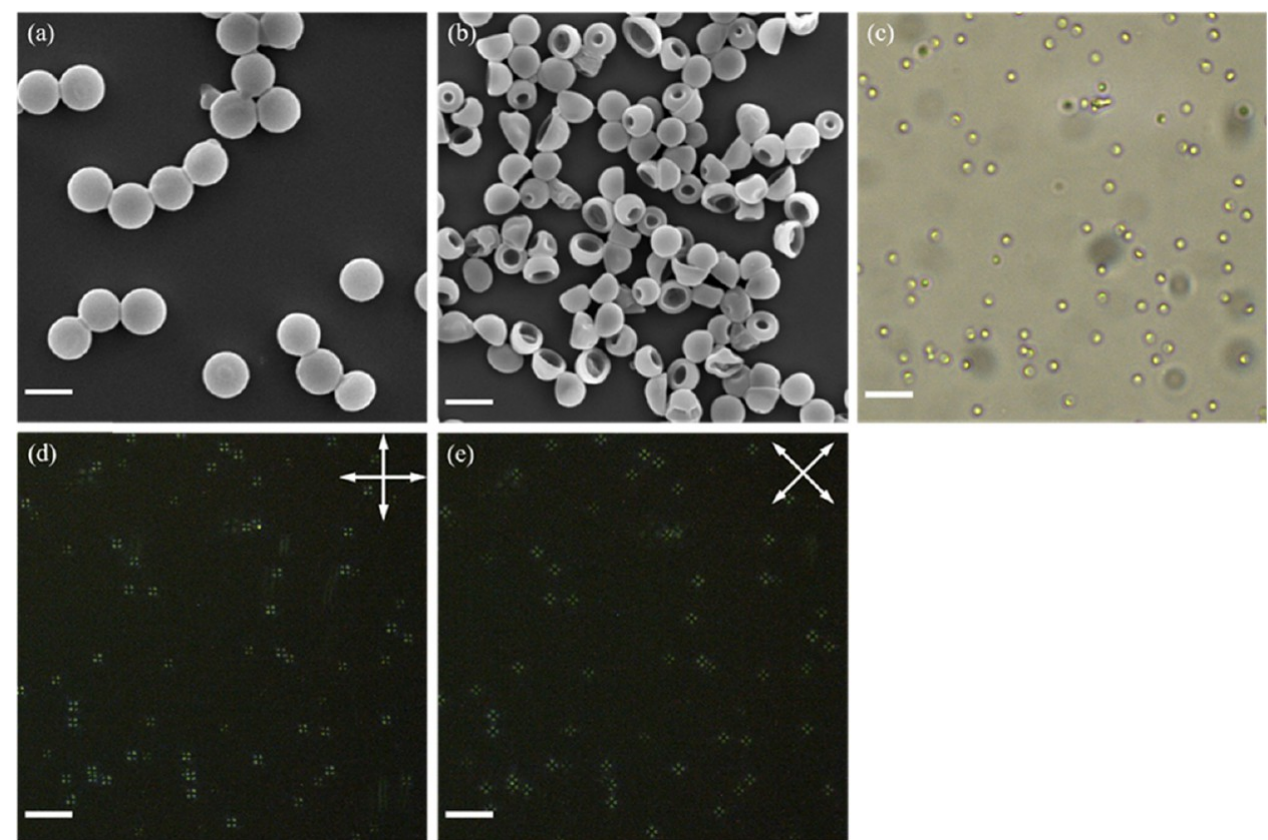

Figure 7. SEM images of (a) core-shell particles and (b) LC shells (scale bar $=2 \mu \mathrm{m}$ ). POM images of LC shells (c) without and (d and e) with cross polarizers (scale bar $=10 \mu \mathrm{m}$ ). The orientation of the cross polarizers is indicated by the arrows. 

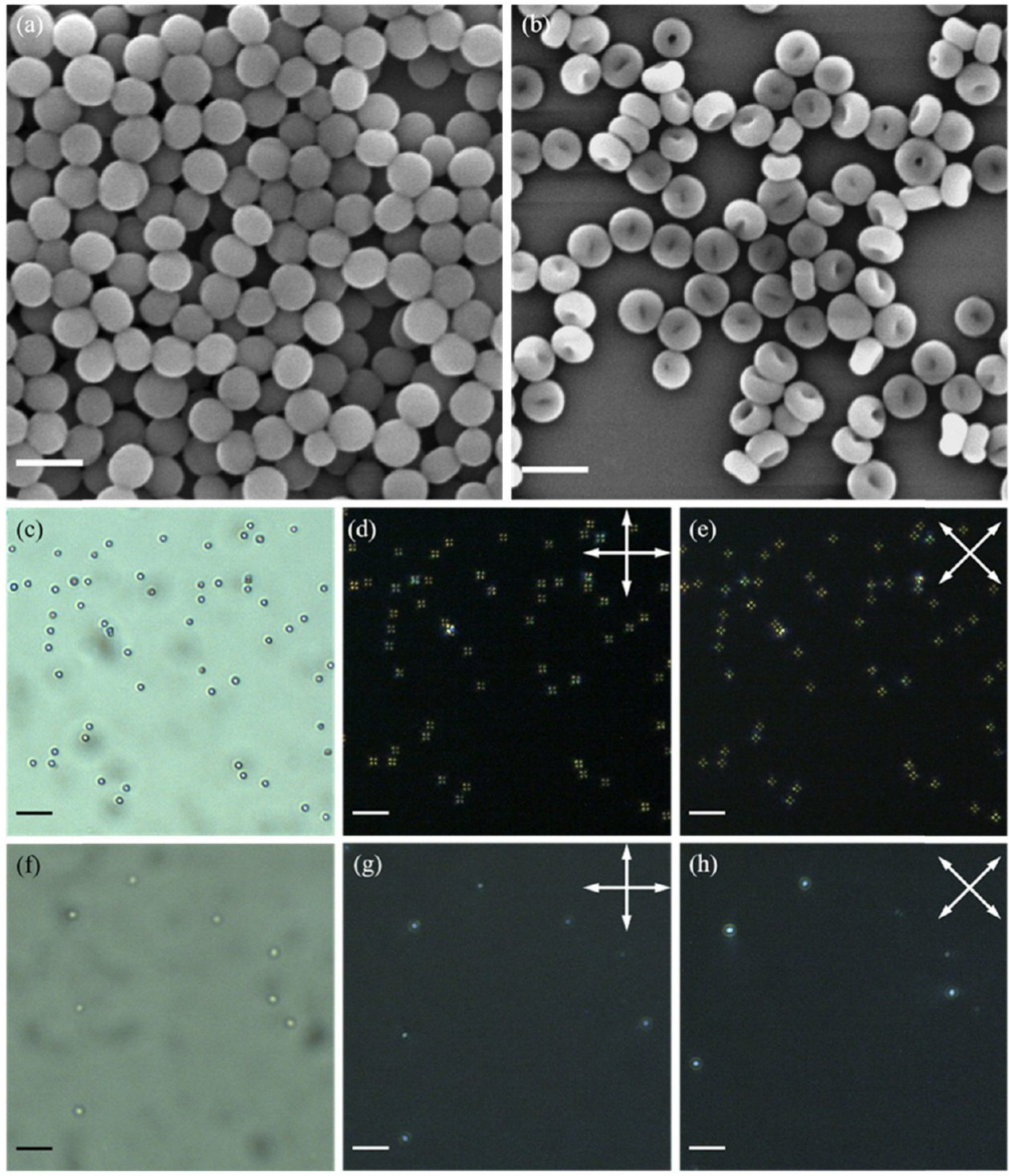

Figure 8. SEM images of the (a) core-shell particles and the (b) shells prepared from monomers 1,2 , and 4 (weight ratio $=20 / 65 / 15)($ scale bar $=2 \mu \mathrm{m}$ ). POM images of the shells (c) without and ( $\mathrm{d}$ and $\mathrm{e}$ ) with crossed polarizers (scale bar $=10 \mu \mathrm{m}$ ); POM images of the shells suspended in glycerol $(\mathrm{f})$ without and $(\mathrm{g}$ and $\mathrm{h}$ ) with crossed polarizers (scale bar $=10 \mu \mathrm{m})$. The orientation of the crossed polarizers is indicated by the arrows.

stable dispersion and particles with low polydispersity were obtained, even in the presence of the carboxylic acidfunctionalized LC monomers. The core-shell particles were smaller than the previous core-shell particles (Table 1, entry 3 ), probably because the yield is lower as the polymerization time is shortened. After THF treatment, a weight loss of $36 \%$ was observed, while the core and shell initially made up 68 and $32 \%$ of the core-shell particle. This indicates that the removal of the seeds is not complete, probably because the diffusion of the polymer chains out of the particles was slowed by the dense LC shell, and harsher conditions are needed to remove the LC seeds more completely. Similar birefringence patterns to those seen in the previous bipolar shells were observed with POM (Figure $8 \mathrm{c}$ to $\mathrm{h}$ ), confirming bipolar alignment in these LC shells, and that effective control on the LC alignment is feasible with the seeded dispersion polymerization method even with more challenging LC monomers.

\section{CONCLUSIONS}

We report the successful preparation of monodisperse, micrometer-sized LC polymer shells via seeded dispersion polymerization. Investigation of the chemical structure of the seeds revealed that with non-LC (PPhMA) seeds, the molecules in the LC polymer shells aligned radially, and shells with small dimples are formed. By polymerizing a monomer mixture with an appropriate crosslinking density in the presence of bipolar LC seeds, hollow bipolar LC polymer shells can be prepared, which collapsed in a biconcave shape. For lower crosslink density LC polymers, cup-shaped polymer shells are formed. LC polymer shells with other functional groups can also be prepared with this method, revealing the versatility of the seeded dispersion polymerization method.

Our findings disclose a new method to fabricate monodisperse LC polymer shells with programmable alignments and shapes. Preliminary results reveal that the azobenzene moieties in the shells can be photoswitched by alternative irradiation of 365 and $455 \mathrm{~nm}$ light (Figure S2), 
which might be interesting for drug delivery and soft actuator applications.

\section{ASSOCIATED CONTENT}

\section{SI Supporting Information}

The Supporting Information is available free of charge at https://pubs.acs.org/doi/10.1021/acs.macromol.1c00884.

Hansen solubility parameter of the liquid monomers and the solubility distance to ethanol and THF; NMR spectrum of the linear LC seeds using monomer 1; and UV-vis data of the LC polymer shells in entry 3 suspended in chlorobenzene after 365 and $455 \mathrm{~nm}$ light irradiation (PDF)

Rotation and flow of LC shells due to the flow of the solvent (Movie S1) (MP4)

\section{AUTHOR INFORMATION}

\section{Corresponding Author}

Albert P.H.J. Schenning - Stimuli-responsive Functional Materials and Devices, Department of Chemical Engineering and Chemistry and Institute for Complex Molecular Systems, Eindhoven University of Technology, 5600 MB Eindhoven, The Netherlands; (1) orcid.org/0000-0002-3485-1984; Email: a.p.h.j.schenning@tue.nl

\section{Authors}

Xiaohong Liu - Stimuli-responsive Functional Materials and Devices, Department of Chemical Engineering and Chemistry and Institute for Complex Molecular Systems, Eindhoven University of Technology, $5600 \mathrm{MB}$ Eindhoven, The Netherlands

Mohammad-Amin Moradi - Institute for Complex Molecular Systems, Eindhoven University of Technology, $5600 \mathrm{MB}$ Eindhoven, The Netherlands; (1) orcid.org/0000-00033754-9200

Tom Bus - Stimuli-responsive Functional Materials and Devices, Department of Chemical Engineering and Chemistry and Institute for Complex Molecular Systems, Eindhoven University of Technology, 5600 MB Eindhoven, The Netherlands

Johan P.A. Heuts - Institute for Complex Molecular Systems, Eindhoven University of Technology, 5600 MB Eindhoven, The Netherlands; Supramolecular Polymer Chemistry Group, Department of Chemical Engineering and Chemistry, Eindhoven University of Technology, $5600 \mathrm{MB}$ Eindhoven,

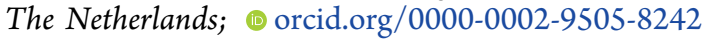

Michael G. Debije - Stimuli-responsive Functional Materials and Devices, Department of Chemical Engineering and Chemistry, Eindhoven University of Technology, $5600 \mathrm{MB}$ Eindhoven, The Netherlands; 0 orcid.org/0000-00018844-1115

Complete contact information is available at:

https://pubs.acs.org/10.1021/acs.macromol.1c00884

\section{Notes}

The authors declare no competing financial interest.

\section{ACKNOWLEDGMENTS}

The authors are grateful for the financial support from The Netherlands Organization for Scientific Research (TOPPUNT 718.016.003).

\section{REFERENCES}

(1) Yu, W.; Chen, Y.; Mao, Z. Hollow Polyelectrolyte Microcapsules as Advanced Drug Delivery Carriers. J. Nanosci. Nanotechnol. 2016, 16, 5435-5446.

(2) Liu, T.; Hu, J.; Ma, X.; Kong, B.; Wang, J.; Zhang, Z.; Guo, D. S.; Yang, X. Hollow Double-Layered Polymer Nanoparticles with SNitrosothiols for Tumor Targeted Therapy. J. Mater. Chem. B 2017, 5, 7519-7528.

(3) Yamala, A. K.; Nadella, V.; Mastai, Y.; Prakash, H.; Paik, P. PolyN-Acryloyl-(1-Phenylalanine Methyl Ester) Hollow Core Nanocapsules Facilitate Sustained Delivery of Immunomodulatory Drugs and Exhibit Adjuvant Properties. Nanoscale 2017, 9, 14006-14014.

(4) Yong, X.; Wu, Y.; Deng, J. Chiral Helical Substituted Polyacetylene Grafted on Hollow Polymer Particles: Preparation and Enantioselective Adsorption towards Cinchona Alkaloids. Polym. Chem. 2019, 10, 4441-4448.

(5) Raza, S.; Yong, X.; Deng, J. Optically Active Biobased Hollow Polymer Particles: Preparation, Chiralization, and Adsorption toward Chiral Amines. Ind. Eng. Chem. Res. 2019, 58, 4090-4098.

(6) Zhou, K.; Tong, L.; Deng, J.; Yang, W. Hollow Polymeric Microspheres Grafted with Optically Active Helical Polymer Chains: Preparation and Their Chiral Recognition Ability. J. Mater. Chem. 2010, 20, 781-789.

(7) Qin, Y.; Wang, L.; Zhao, C.; Chen, D.; Ma, Y.; Yang, W. Ammonium-Functionalized Hollow Polymer Particles As a $\mathrm{PH}$ Responsive Adsorbent for Selective Removal of Acid Dye. ACS Appl. Mater. Interfaces 2016, 8, 16690-16698.

(8) Wang, X.; Ji, H.; Zhang, X.; Zhang, H.; Yang, X. Hollow Polymer Microspheres Containing a Gold Nanocolloid Core Adsorbed on the Inner Surface as a Catalytic Microreactor. J. Mater. Sci. 2010, 45, 3981-3989.

(9) Tian, Q.; Yu, X.; Zhang, L.; Yu, D. Monodisperse Raspberry-like Multihollow Polymer/Ag Nanocomposite Microspheres for Rapid Catalytic Degradation of Methylene Blue. J. Colloid Interface Sci. 2017, 491, 294-304.

(10) Li, X.; Cai, T.; Kang, E. T. Hairy Hybrid Nanorattles of Platinum Nanoclusters with Dual-Responsive Polymer Shells for Confined Nanocatalysis. Macromolecules 2016, 49, 5649-5659.

(11) Fleischmann, E. K.; Liang, H. L.; Kapernaum, N.; Giesselmann, F.; Lagerwall, J.; Zentel, R. One-Piece Micropumps from Liquid Crystalline Core-Shell Particles. Nat. Commun. 2012, 3, 1178.

(12) Fleischmann, E.-K.; Liang, H.-L.; Lagerwall, J.; Zentel, R. Towards Micrometer Sized Core-Shell Actuators from Liquid Crystalline Elastomers by a Continuous Flow Synthesis. Emerg. Liq. Cryst. Technol. VII 2012, 8279, No. 82790M.

(13) Jampani, V. S. R.; Mulder, D. J.; De Sousa, K. R.; Gélébart, A. H.; Lagerwall, J. P. F.; Schenning, A. P. H. J. Micrometer-Scale Porous Buckling Shell Actuators Based on Liquid Crystal Networks. Adv. Funct. Mater. 2018, 28, No. 1801209.

(14) Jampani, V. S. R.; Volpe, R. H.; De Sousa, K. R.; Machado, J. F.; Yakacki, C. M.; Lagerwall, J. P. F. Liquid Crystal Elastomer Shell Actuators with Negative Order Parameter. Sci. Adv. 2019, 5, No. eaaw2476.

(15) Vennes, M.; Martin, S.; Gisler, T.; Zentel, R. Anisotropic Particles from LC Polymers for Optical Manipulation. Macromolecules 2006, 39, 8326-8333.

(16) Vennes, M.; Zentel, R. Liquid-Crystalline Colloidal Particles. Macromol. Chem. Phys. 2004, 205, 2303-2311.

(17) Liu, X.; Xu, Y.; Heuts, J. P. A.; Debije, M. G.; Schenning, A. P. H. J. Monodisperse Liquid Crystal Network Particles Synthesized via Precipitation Polymerization. Macromolecules 2019, 52, 8339-8345.

(18) Okubo, M.; Fujibayashi, T.; Yamada, M.; Minami, H. MicronSized, Monodisperse, Snowman/Confetti-Shaped Polymer Particles by Seeded Dispersion Polymerization. Colloid Polym. Sci. 2005, 283, 1041-1045.

(19) Wang, R. K.; Liu, H. R.; Wang, F. W. Facile Preparation of Raspberry-like Superhydrophobic Polystyrene Particles via Seeded Dispersion Polymerization. Langmuir 2013, 29, 11440-11448. 
(20) Stefanis, E.; Panayiotou, C. Prediction of Hansen Solubility Parameters with a New Group-Contribution Method. Int. J. Thermophys. 2008, 29, 568-585.

(21) Haseloh, S.; Zentel, R. Synthesis of Liquid-Crystalline Colloids in Nonpolar Media and Their Manipulation in Electric Fields. Macromol. Chem. Phys. 2009, 210, 1394-1401.

(22) Song, J. S.; Chagal, L.; Winnik, M. A. Monodisperse Micrometer-Size Carboxyl-Functionalized Polystyrene Particles Obtained by Two-Stage Dispersion Polymerization. Macromolecules 2006, 39, 5729-5737. 